\title{
Association between polymorphisms in the promoter region of $T$ cell immunoglobulin and mucin domain-3 and myasthenia gravis-associated thymoma
}

\author{
GUOWU XU $^{1 *}, \mathrm{KAI} \mathrm{ZHENG}^{2^{*}}, \mathrm{XING} \mathrm{LU}^{3}$, JINXIANG WANG ${ }^{1}$, YANFEN CHAI ${ }^{1}$ and JUNYI WANG ${ }^{3}$ \\ Departments of ${ }^{1}$ Emergency and ${ }^{2}$ Cardiothoracic Surgery, Tianjin Medical University General Hospital, Tianjin \\ 300052; ${ }^{3}$ Department of Intensive Care Unit, Tianjin Third Central Hospital, Tianjin 300170, P.R. China
}

Received March 27, 2014; Accepted December 12, 2014

DOI: $10.3892 / \mathrm{ol} .2015 .2845$

\begin{abstract}
Thymoma is a type of benign or low-grade malignant tumor, occurring on the thymic epithelium. Patients with thymoma may also suffer from myasthenia gravis (MG), presenting MG-associated thymoma. T cell immunoglobulin and mucin domain-3 (Tim-3), a subtype of the Tim protein family, may be an important immune regulatory and pivotal molecule associated with tumor development. In order to understand the etiology and pathogenesis of MG-associated thymoma in the Han population of North China, the present study investigated the association between a polymorphism on the -574 locus in the promoter of Tim-3 and the risk of MG-associated thymoma in the Han Chinese population. In total, 116 patients with thymoma and MG were enrolled into the MG-associated thymoma group, while 124 patients with thymoma, but without MG, were enrolled into the non-MG-associated thymoma group. Examinations were conducted to reach a definite diagnosis of thymoma and MG and rule out other autoimmune diseases. Allele-specific polymerase chain reaction (AS-PCR) was performed to determine the polymorphism on the -574 locus of Tim-3 in all the subjects. PCR products were randomly selected for sequencing. Statistically significant differences were detected between the distribution frequencies of the GT+TT genotype and T allele on the -574 locus of the MG-associated thymoma group (31.03 vs. $12.90 \%$, respectively;
\end{abstract}

Correspondence to: Professor Kai Zheng, Department of Cardiothoracic Surgery, Tianjin Medical University General Hospital, 154 Anshan Road, Tianjin 300052, P.R. China

E-mail: zhkeith@163.com

*Contributed equally

Abbreviations: AS-PCR, allele-specific polymerase chain reaction; $\mathrm{CI}$, confidence interval; EAE, experimental autoimmune encephalomyelitis; MG, myasthenia gravis; MS, multiple sclerosis; OR, odds ratio; Tim, T-cell immunoglobulin and mucin domain.

Key words: thymoma, myasthenia gravis, T-cell immunoglobulin and mucin domain-3, gene polymorphism $\left.\chi^{2}=11.609, \mathrm{P}=0.001\right)$ and the non-MG-associated thymoma group ( 15.52 vs. $6.45 \%$, respectively; $\left.\chi^{2}=10.198, \mathrm{P}=0.001\right)$. In conclusion, the present study indicated that an association may exist between the polymorphism of the -574 locus in the Tim- 3 promoter and MG-associated thymoma.

\section{Introduction}

Thymoma is a type of benign or low-grade malignant tumor, developed on the thymic epithelium, and the most frequent anterior mediastinal neoplasm in adults; however, its overall incidence is only 0.13 per 100,000 individuals/year according to data from the Surveillance, Epidemiology and End Results database (1-3). Due to the low incidence of this neoplasm, its etiology and pathogenesis remain unknown. In addition, epidemiological data have revealed that $20-25 \%$ of patients with thymomas also suffer from myasthenia gravis (MG; hereafter referred to as MG-associated thymoma), whereas $10-20 \%$ of myasthenic patients suffer from thymomas (4). MG, is an autoimmune disease of unclear etiology, results in even more complex etiology and pathogenesis of MG-associated thymoma. The ratio of patients with thymoma and $\mathrm{MG}$ is higher than that of any ofther autoimmune disease. Pivotal markers and cross-linked molecules of thymoma and MG have been the aim of several previous studies $(5,6)$.

Thymus is a primary organ and the initial site for the development of the $\mathrm{T}$ cell immunological function (7). The development of thymoma is frequently accompanied by a rich infiltrate of $\mathrm{T}$ cells (3). When released into the circulation, these abnormally conditioned $\mathrm{T}$ cells are hypothesized to be responsible for the autoimmune conditions that often accompany thymoma, particularly MG (3). MG is a known prototypical $\mathrm{CD}^{+} \mathrm{T}$ cell-dependent autoimmune disease. Various subsets of $\mathrm{T}$ helper (Th) cells, including Th1, Th2 and Th17, and regulatory $\mathrm{T}$ cells (Treg) have been suggested to be involved in the pathogenesis of MG (8). In addition, the imbalance of different $\mathrm{Th}$ cell subsets is important in the progression of the disease. Thymoma and MG are multifactorial and noninherited diseases, with an established genetic predisposition. Numerous studies have demonstrated that certain human leukocyte antigen (HLA) and non-HLA genes, including HLA-death receptor 3, PTPN22 
and CTLA4, present an evident association with MG (9). However, a clear correlation of particular genes/molecules with MG-associated thymoma cannot be gained from the limited present research (10).

$\mathrm{T}$ cell immunoglobulin and mucin domain-3 (Tim-3) is a subtype of the Tim protein family, which was initially identified in 2002, and is selectively expressed on Th1 cells, but not on Th2 cells (11). Tim-3 has been demonstrated to negatively regulate Th1 response and induce immune tolerance through the Tim-1/galectin-9 signaling pathway in autoimmune diseases (12). Furthermore, suppression of Tim-3 expression has been revealed to enhance the pathological severity of experimental autoimmune encephalomyelitis (EAE) (13), while Tim-3-deficient mice have been found to be refractory to the induction of immune tolerance in EAE (14). Besides the important role of Tim-3 in autoimmune diseases, a recent study has indicated that Tim-3 is also a molecular switch for tumor escape from innate immunity (15). Previous studies have further identified Tim-3 expression on exhausted $\mathrm{T}$ cells in human tumors $(16,17)$ and preclinical tumor models $(18,19)$. The expression of Tim-3 is significantly increased on T cells in tumor-infiltrated tissues and on tumor-infiltrating lymphocytes in peripheral lymphoid tissues or the blood of tumor patients, indicating that Tim-3 may be upregulated in a tumor-derived environment $(17,18)$. Therefore, the present study hypothesized that Tim-3 may be a potentially pivotal molecule in autoimmune disease-associated tumors, such as MG-associated thymoma.

Tim-3 polymorphism has been demonstrated to alter the interaction between Tim-3 and its ligand, thereby affecting the process that results in certain immune diseases $(19,20)$ and being actively involved in the pathogenesis of tumors (21). Considering the aforementioned findings, Tim-3 appears to be an important regulatory molecule that plays a critical role in MG-associated thymoma, which may be triggered mainly by the deviation of Th cell subtypes. However, to the best of our knowledge, Tim-3 polymorphism and its association with MG-associated thymoma in the Han Chinese population of North China have not been evaluated. The aim of the present study was to investigate the polymorphism of the -574 locus in the promoter of Tim-3 and its association with MG-associated thymoma in the Han Chinese population of North China.

\section{Materials and methods}

Study approval. The experiments of this study were approved by the Ethics Committee of the Tianjin Medical University General Hospital (Tianjin, China). Written informed consent was obtained from all the patients prior to participation in the study. All the subjects were from the Han population of North China, since the study was performed at Tianjin, China.

Patients and DNA samples. In total, 116 patients with thymoma and MG were enrolled from the Department of Cardiothoracic Surgery of the Tianjin Medical University General Hospital, comprising the study group (MG-associated thymoma group). In addition, 124 patients with thymoma, but without MG, were randomly selected from the Department of Cardiothoracic Surgery of the Tianjin Medical University General Hospital, forming the control group (non-MG-associated thymoma group). All the subjects were subjected to a comprehensive examination in order to obtain a definite diagnosis of thymoma and MG and exclude other autoimmune diseases. For the diagnosis of thymoma, the results of chest X-ray, computed tomography and magnetic resonance imaging, as well as surgical and pathological findings, were considered. The diagnostic criteria of MG included myasthenic manifestation, neostigmine test, repetitive nerve stimulation test and serum autoantibody detection. All the patients included in this study were genetically unrelated individuals of the Han Chinese population. Genomic DNA was extracted from the white blood cells of each sample using a DNA isolation kit (GK1072, Generay Biotech Co, Ltd., Shanghai, China), according to the manufacturer's instructions. The extracted genomic DNA was dissolved in sterile double-distilled water. Subsequently, the concentrations and absorbance ratios at $260 \mathrm{~nm} / 280 \mathrm{~nm}$ (A260/A280) of the DNA solutions were measured using a nucleic acid spectrometer (ScanDrop 200; Analytik Jena AG, Jena, Germany). DNA samples with A260/A280 ratios ranging between 1.7 and 2.0 were selected as polymerase chain reaction (PCR) templates and stored at $-80^{\circ} \mathrm{C}$.

-574 locus genotyping. Allele-specific PCR (AS-PCR) was used to investigate the -574 locus and three primers were designed using Primer 5.0 software (Premier Biosoft, Palo Alto, CA,USA), according to the flanking sequence (GenBank no. NM_032782). The primer sequences were as follows: Forward 1 (F1), 5'-GGC TTATGCTGGGAGTTGCT-3'; forward 2 (F2), 5'-GGCTTA TGCTGGGAGTTGCG-3'; reverse for F1 and F2 (R), 5'-GGT GTCTGATTGCCAGTGATTC-3'. The F1 and R primers were used to amplify $\mathrm{T}$ allele fragments, whereas $\mathrm{F} 2$ and $\mathrm{R}$ primers were used to amplify $G$ allele fragments. All the amplified fragments were 539 bp long and each sample was subjected to AS-PCR with F1/R and F2/R. The reaction mixture was as follows: $2.5 \mu \mathrm{l} 10 \mathrm{X}$ PCR buffer; $1.5 \mu \mathrm{l}$ deoxyribonucleotide triphosphate (2.5 mmol/1 each); $1 \mu \mathrm{l}$ forward primer (5 $\mu \mathrm{mol} / \mathrm{l})$; $1 \mu \mathrm{l}$ reverse primer $(5 \mu \mathrm{mol} / \mathrm{l}) ; 1.0 \mu \mathrm{l} \mathrm{DNA}$ template; and $0.5 \mu \mathrm{l}$ Taq DNA polymerase $(2.5 \mathrm{U} / \mu \mathrm{l})$ (Beijing AuGCT DNA-Syn Biotechnology Co., Ltd., Beijing, China). Double-distilled water was added to obtain a final reaction volume of $25 \mu 1$ and, subsequently, touchdown PCR was performed to amplify the target amplicons. The reaction mixture was heated in a Thermo Cycler (Mastercycler 5333; Eppendorf AG, Hamburg, Germany) under the following conditions: $95^{\circ} \mathrm{C}$ for $5 \mathrm{~min} ; 94^{\circ} \mathrm{C}$ for $30 \mathrm{sec}$; progressive lowering of the annealing temperature by $1^{\circ} \mathrm{C}$ every three $30 \mathrm{sec}$ annealing cycles (between $65^{\circ} \mathrm{C}$ and $56^{\circ} \mathrm{C}$ ); extension at $65^{\circ} \mathrm{C}$ for $35 \mathrm{sec}$, followed by 30 cycles at $72^{\circ} \mathrm{C}$ for $35 \mathrm{sec}$; final extension at $72^{\circ} \mathrm{C}$ for $10 \mathrm{~min}$. The amplified products were subjected to $1.8 \%$ agarose gel electrophoresis and visualized using ethidium bromide staining, followed by capturing images using FireReader software (XS D5626M Auto + FC26WL + Uviband; UVItec Ltd., Cambridge, UK).

DNA sequencing. Several PCR products were randomly selected and purified using a PCR purification kit (DK004-01B; NovoProtein Biotechnology Co., Ltd., Shanghai, China). Sequencing of the purified products was performed by Beijing AuGCT DNA-SYN Biotechnology Co., Ltd. (Beijing, China).

Statistical analyses. Statistical analysis was performed using the SPSS 17.0 software package (SPSS, Inc., Chicago, IL, 
Table I. Distribution of -574 locus of Tim-3 genotypes of the studied population (\%).

\begin{tabular}{|c|c|c|c|c|c|c|}
\hline Genotype & $\begin{array}{l}\text { Study group } \\
(\mathrm{n}=116), \mathrm{n}(\%)\end{array}$ & $\begin{array}{l}\text { Control group } \\
(n=124), n(\%)\end{array}$ & $\chi^{2}$-value & $\mathrm{P}$-value & OR & $95 \% \mathrm{CI}$ \\
\hline GG & $80(68.97)$ & $108(87.10)$ & 11.609 & 0.001 & 0.329 & $0.171-0.634$ \\
\hline $\mathrm{GT}+\mathrm{TT}$ & $36+0(31.03)$ & $16+0(12.90)$ & & & & \\
\hline
\end{tabular}

Tim-3, T cell immunoglobulin and mucin domain-3; OR, odds ratio; CI, confidence interval.

Table II. Distribution of different alleles on the -574 locus.

\begin{tabular}{lccccc}
\hline Alleles & $\begin{array}{c}\text { Study group } \\
(\mathrm{n}=116), \mathrm{n}(\%)\end{array}$ & $\begin{array}{c}\text { Control group } \\
(\mathrm{n}=124), \mathrm{n}(\%)\end{array}$ & $\chi^{2}$-value & P-value & OR \\
\hline $\mathrm{G}$ & $196(84.48)$ & $232(93.55)$ & 10.198 & 0.001 & 0.375 \\
$\mathrm{~T}$ & $36(15.52)$ & $16(6.45)$ & & & $0.202-0.697$ \\
\hline
\end{tabular}

USA). $\chi^{2}$ test was performed to compare the allele frequencies of each group, while the Hardy-Weinberg equilibrium test was conducted to investigate the demographic representation of the study and control groups. The relative risk was presented as the odds ratio (OR) and its $95 \%$ confidential interval (CI). $\mathrm{P}<0.05$ was considered to indicate a statistically significant difference.

\section{Results}

General characteristics. The ages of the MG-associated thymoma patients were 18-62 years with a mean age of $45.6 \pm 4.5$ years, while the ages of the non-MG-associated thymoma patients were 20-63 years with a mean age of $46.4 \pm 4.3$ years. No statistically significant difference was observed between the mean ages of the two patient groups ( $P>0.05)$. In addition, the Hardy-Weinberg equilibrium test results for the -574 locus revealed no statistically significant differences between the study and control groups $(\mathrm{P}>0.05)$, which indicated that the two groups were in Hardy-Weinberg equilibrium and demographically representative.

-574 locus. At the -574 locus, the $\mathrm{G}$ and $\mathrm{T}$ alleles were defined as wild-type and mutant alleles, respectively. Following agarose gel electrophoresis, the GG genotypes were defined as wild GG homozygotes, the TT genotypes were defined as mutant TT homozygotes and the GT genotypes were defined as GT heterozygotes (Fig. 1). In the present study, the GG genotype was identified in $80 \mathrm{MG}$-associated thymoma patients, while the GT genotype was identified in the remaining $36 \mathrm{MG}$-associated thymoma patients. By contrast, the GG genotype was detected in 108 non-MG-associated thymoma patients, while the GT genotype was detected in the remaining 16 patients of the group. The TT genotype was not identified in the study or control patients (Figs. 2 and 3).

Genotypes and frequencies. At the -574 locus of Tim-3 in the MG-associated group, the GG and (GT+TT) genotypes presented frequencies of 68.97 and $31.03 \%$, respectively. By contrast, $87.10 \%$ of the control subjects possessed the

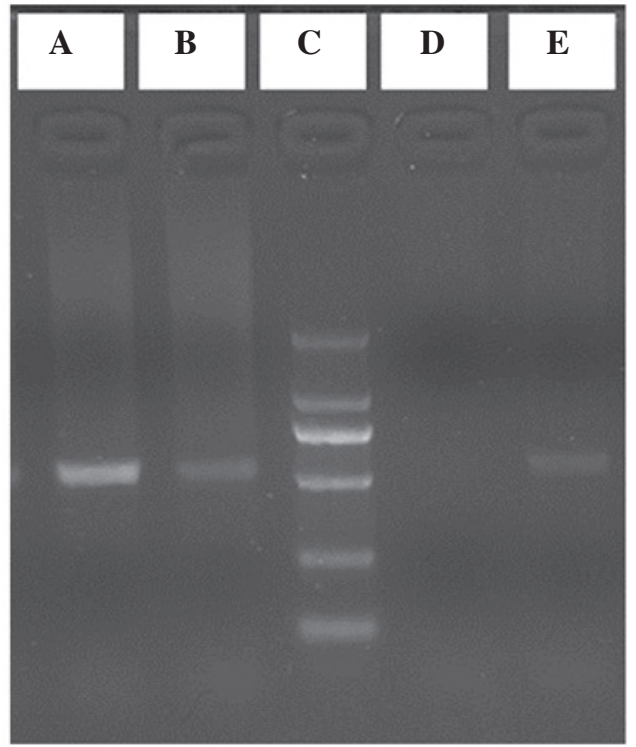

Figure 1. Electrophoretogram of -574 locus in the Tim-3 gene. A and B represent the TG genotype; C represents the DNA marker with 2000, 1000, 750, 500, 250 and $100 \mathrm{bp}$; D and E represent the GG genotype. Tim-3, T cell immunoglobulin and mucin domain-3;

GG genotype and the remaining $12.90 \%$ possessed the (GT+TT) genotype. A statistically significant difference was observed between the two groups $\left(\chi^{2}=11.609, \mathrm{P}=0.001\right)$. The frequency of the GT+TT genotype on the -574 locus of the MG-associated thymoma group was found to be statistically different compared with the control group $(\mathrm{P}=0.001$, $\mathrm{OR}=0.329$; Table I). In addition, the frequencies of $\mathrm{T}$ allele on the -574 loci of the two groups were statistically different $(\mathrm{P}=0.001, \mathrm{OR}=0.375$; Table II $)$.

\section{Discussion}

Tim-3, a surface molecule on $\mathrm{T}$ cells, is important in immune regulatory functions and presents a strong correlation with various tumor types. In humans, Tim-3 is located 


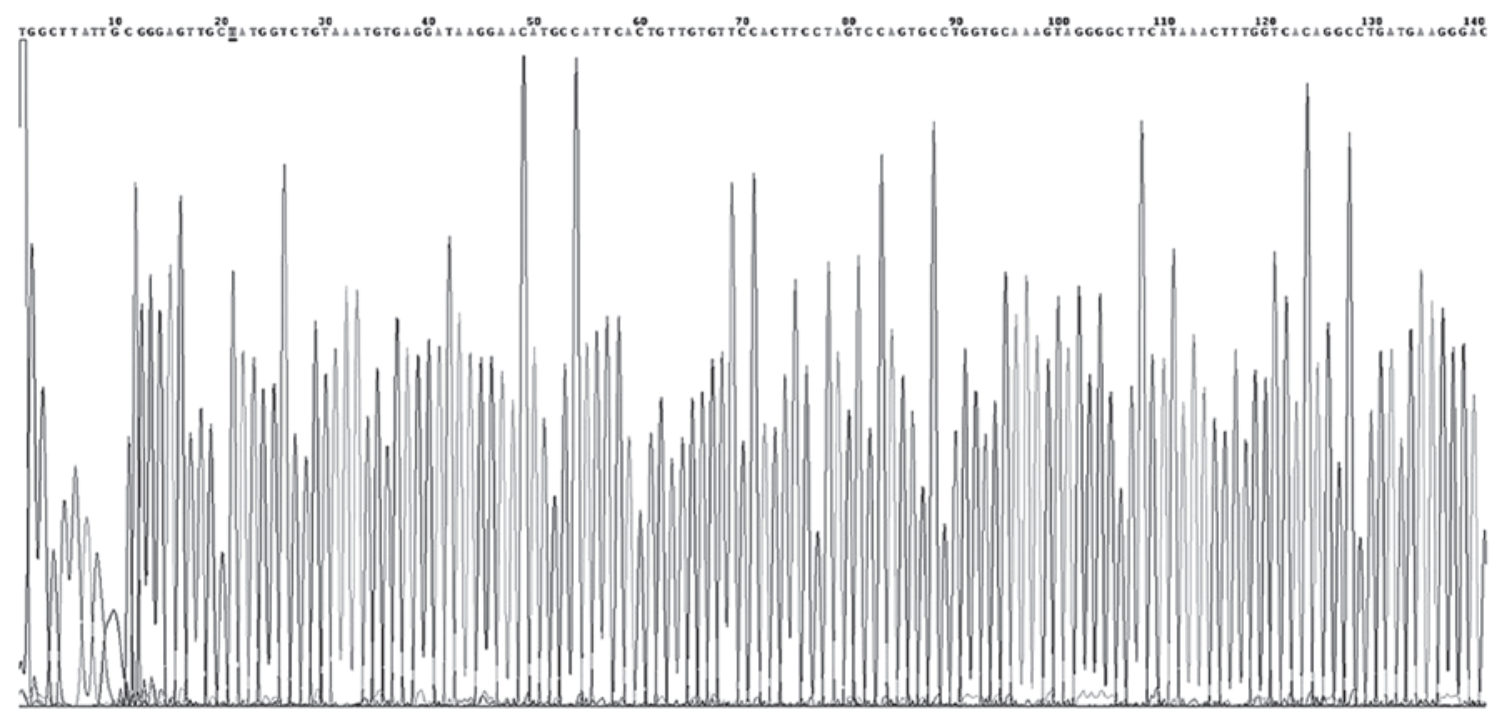

Figure 2. Sequence diagram of $\mathrm{T}$ allele amplified product at the -574 locus of the Tim-3 promoter region. $\mathrm{T}$ allele is underlined.

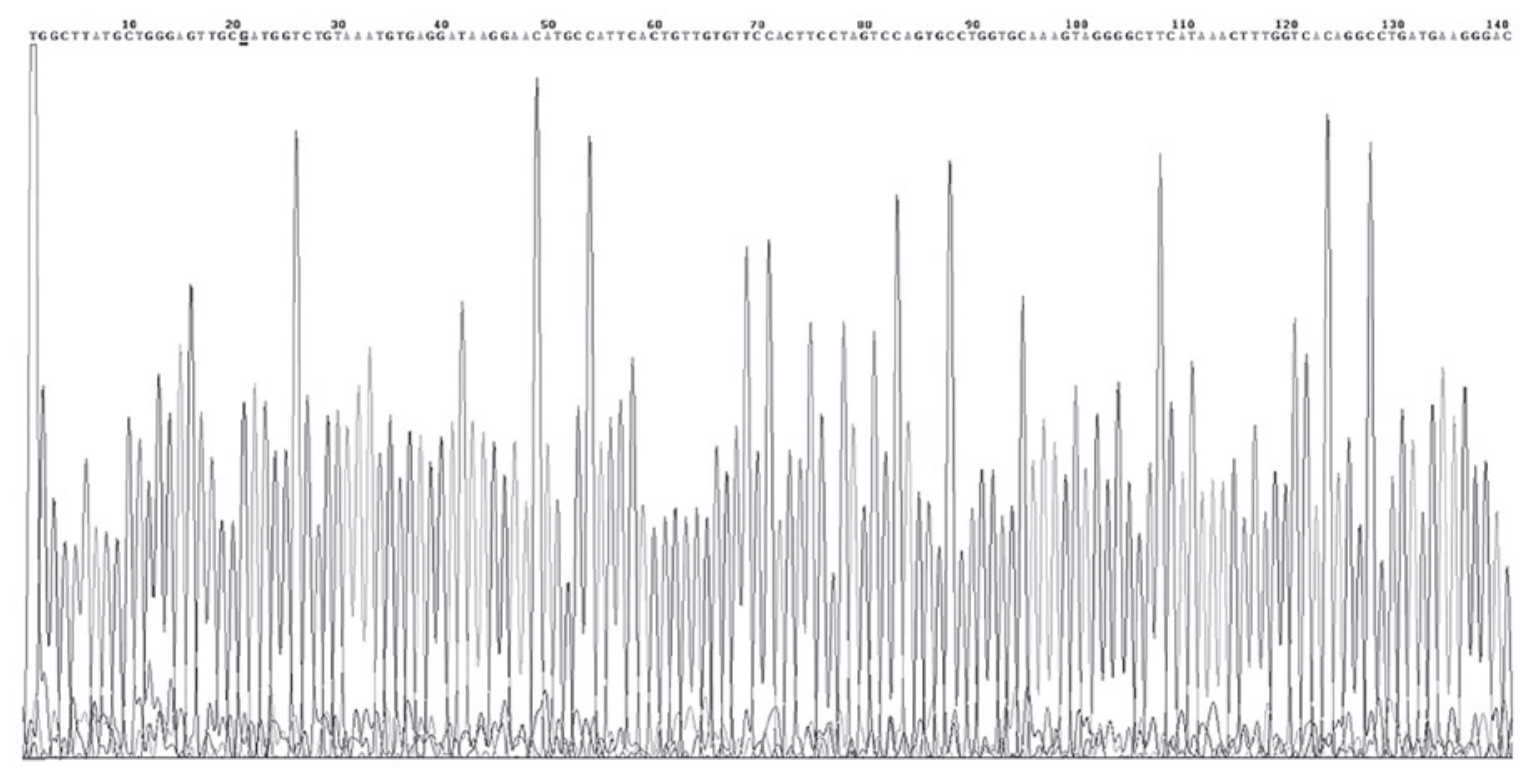

Figure 3. Sequence diagram of $\mathrm{G}$ allele amplified product at the -574 locus of the Tim-3 promoter region. $\mathrm{G}$ allele is underlined.

on chromosome 5q33.2 and comprises 301 amino acids, including elementary structural domains, such as the signal domain, mucin-like structural domain, transmembrane zone and cytomere domain of the phosphorylation site $(13-17,20)$. In the immunoglobulin $\mathrm{V}$ structural domain, two antiparallel $\beta$ fragments and a metal ion ligand binding site function together as the ligand-binding site of Tim-3 (14,23).

Evidence indicates that Tim-3 is a negative regulator of $\mathrm{T}$ cell responses and is involved in the modulation of autoimmune diseases (20). Identification of galectin-9 as a ligand of Tim-3 may induce the death of Th1 cells and downregulate Th1 responses $(12,23)$. A previous study demonstrated that in vivo treatment with Tim-3 monoclonal antibodies during the induction of EAE, which is a mouse model of multiple sclerosis (MS), accelerated disease progression (11). In human patients with MS, T cell clones derived from the cerebrospinal fluid expressed lower levels of Tim-3 compared with those from healthy control subjects (24). A study on a mouse model of autoimmune diseases has indicated that inhibition of the interaction between Tim-3 and its ligand may dramatically aggravate the manifestation of autoimmune diseases (25). Furthermore, the distribution frequencies of $+4259 \mathrm{~T} / \mathrm{G}$ in Tim-3 in patients with rheumatoid arthritis have been demonstrated to be statistically different compared with healthy individuals in the Han and Hui Chinese populations (26). Therefore, the present study hypothesized that Tim-3 may be a protective factor of autoimmune diseases (it may protect patients from suffering from autoimmune diseases or decrease the possibility of suffering from autoimmune diseases), while Tim-3 polymorphisms may be closely associated with autoimmune diseases.

The role of Tim-3 in tumor tissues has been investigated in numerous studies. Piao et al (27) identified that Tim-3 expression was higher in prostate cancer tissues compared with the 
adjacent benign tissues. In addition, Cao et al (28) revealed that Tim-3 expression in cervical cancer promoted tumor metastasis. Furthermore, a number of studies identified that the distribution frequencies of $+4259 \mathrm{~T} / \mathrm{G}$ in Tim-3 in patients with pancreatic cancer or renal cell carcinoma were statistically different compared with healthy individuals $(29,30)$. A recent study also demonstrated that, following tumor-associated expression of the receptor Tim-3 by dendritic cells, Tim-3 inhibited the antitumor efficacy of DNA vaccines and chemotherapy (31). Therefore, Tim-3 may be a risk factor of tumorigenesis, while Tim-3 polymorphisms may be associated with cancer.

In the present study, polymorphisms at the -574 locus of Tim-3 were investigated. Statistical analysis revealed that, in the MG-associated and non-MG-associated thymoma groups, the mutant-type homozygote TT frequencies were zero. Thus, the mutant-type homozygote TT and heterozygote GT phenotypes were merged and reanalyzed using the $\chi^{2}$ test. The results detected a statistically significant difference in the distribution frequencies of the GT+TT genotype between the two groups (Table I). In addition, the distribution frequencies of $\mathrm{T}$ allele on the -574 locus were significantly different between the two groups (Table II). These findings indicated an association between the -574 locus polymorphism and MG-associated thymoma in the Han population of North China. Furthermore, the OR values were found to be 0.329 and 0.375 for the distribution frequencies of the GT+TT genotype and T allele, respectively, on the -574 locus between the two groups. Based on the results of the aforementioned studies, Tim-3 was hypothesized to be a protective factor in autoimmune diseases and a risk factor of tumorigenesis; however, to date, the association between tumorigenesis and autoimmune diseases remains unclear. Thus, the present study investigated the correlation between Tim-3 and MG-associated thymoma, which is a tumor commonly associated with autoimmune diseases. In conclusion, Tim-3 was found to be a protective factor in MG-associated thymoma, indicating that thymoma is affected by MG. Therefore, future research on thymoma should investigate the possible association with MG.

\section{Acknowledgements}

This study was supported by a grant from the Tianjin Medical University General Hospital. The authors would like to thank Dr Wang Beilei (Endocrine Laboratory, Tianjin Medical University, Tianjin, China) for her technical assistance.

\section{References}

1. Priola AM and Priola SM: Imaging of thymus in myasthenia gravis: from thymic hyperplasia to thymic tumor. Clin Radiol 65: e230-e245, 2014.

2. Shapiro M and Korst RJ: Surgical approaches for stage IVA thymic epithelial tumors. Front Oncol 3: 332, 2014.

3. Engels EA: Epidemiology of thymoma and associated malignancies. J Thorac Oncol 5 (10 Suppl 4): S260-S265, 2010.

4. Lucchi M, Ricciard R, Melfi F, et al: Association of thymoma and myasthenia gravis: oncological and neurological results of the surgical treatment. Eur J Cardiothorac Surg 35: 812-816, 2009.

5. Yu L, Zhang XJ, Ma S, et al: Different characteristics of thymomas with and without myasthenia gravis. Ann Surg Oncol 19: 94-98, 2012.

6. Zheng K, Xu G, Lu X, et al: Expression and polymorphisms of $\mathrm{T}$ cell immunoglobulin domain and mucin domain protein-1 in thymoma with or without myasthenia gravis. Oncol Lett 8: 317-322, 2014
7. Pearse G: Normal structure, function and histology of the thymus. Toxicol Pathol 34: 504-514, 2006.

8. Wang Z, Wang W, Chen Y and Wei D: T helper type 17 cells expand in patients with myasthenia-associated thymoma. Scand J Immunol 76: 54-61, 2012.

9. Zagoriti Z, Kambouris ME, Patrinos GP, et al: Recent advance in genetic predisposition of myasthenia gravis. Biomed Res Int 2013: 404053, 2013.

10. Zheng K, Zhang J, Guo Y and Zhang P: Expression and clinical significance of protein tyrosine phosphatase nonreceptor 22 in resected thymoma. Clin Lab 59: 1041-1044, 2013.

11. Monney L, Sabatos CA, Gaglia JL, et al: Th1-specific cell surface protein Tim-3 regulates macrophage activation and severity of an autoimmune disease. Nature 415: 536-541, 2002.

12. Zhu C, Anderson AC, Schubart A, et al: The Tim-3 ligand galectin-9 negatively regulates $\mathrm{T}$ helper type 1 immunity. Nat Immunol 6: 1245-1252, 2005.

13. Anderson AC and Anderson DE: TIM-3 in autoimmunity. Curr Opin Immunol 18: 665-669, 2006.

14. Sabatos CA, Chakravarti S, Cha E, et al: Interaction of Tim-3 and Tim-3 ligand regulates T helper type 1 responses and induction of peripheral tolerance. Nat Immunol 4: 1102-1110, 2003.

15. Mattei F and Schiavoni G: TIM-3 as a molecular switch for tumor escape from innate immunity. Front Immunol 3: 418, 2013.

16. Fourcade J, Sun Z, Benallaoua M, et al: Upregulation of Tim-3 and PD-1 expression is associated with tumor antigen-specific $\mathrm{CD}^{+} \mathrm{T}$ cell dysfunction in melanoma patients. J Exp Med 207: 2175-2186, 2010.

17. Baitsch L, Baumgaertner P, Devêvre E, et al: Exhaustion of tumor-specific $\mathrm{CD} 8^{+} \mathrm{T}$ cells in metastases from melanoma patients. J Clin Inverst 121: 2350-2360, 2011.

18. Sakuishi K, Apetoh L, Sullivan JM, et al: Targeting Tim-3 and PD-1 pathways to reverse T cell exhaustion and restore anti-tumor immunity. J Exp Med 207: 2187-2194, 2010.

19. Zhou Q, Munger ME, Veenstra RG, et al: Coexpression of Tim-3 and PD-1 identifies a CD8 ${ }^{+}$T-cell exhaustion phenotype in mice with disseminated acute myelogenous leukemia. Blood 117 : 4501-4510, 2011.

20. Mclntire JJ, Umetsu SE, Macaubas C, et al: Immunology: hepatitis A virus link to atopic disease. Nature 425: 576, 2003.

21. Shen Y, Wang C, Hong D, et al: The relationship between polymorphisms in the promoter region of Tim-3 and unexplained recurrent spontaneous abortion in Han Chinese women. Reprod Biol Endocrinol 11: 104, 2013.

22. Shang Y, Li Z, Li H, et al: TIM-3 expression in human osteosarcoma: Correlation with the expression of epithelial-mesenchymal transition-specific biomarkers. Oncol Lett 6: 490-494, 2013.

23. Freeman GJ, Casasnovas JM, Umetsu DT and DeKruyff RH: TIM genes: a family of cell surface phosphatidylserine receptors that regulate innate and adaptive immunity. Immunol Rev 235: $172-189,2010$.

24. Koguchi K, Anderson DE, Yang L, et al: Dysregulated T cell expression of TIM3 in multiple sclerosis. J Exp Med 203: 1413-1418, 2006.

25. Meyers JH, Sabatos CA, Chakravarti S and Kuchroo VK: The TIM gene family regulates autoimmune and allergic diseases. Trends Mol Med 11: 362-369, 2005.

26. Xu J, Yang Y, Liu X and Wang Y: The $-1541 \mathrm{C}>\mathrm{T}$ and $+4259 \mathrm{G}>\mathrm{T}$ of TIM-3 polymorphisms are associated with rheumatoid arthritis susceptibility in a Chinese Hui population. Int $\mathrm{J}$ Immunogenet 38: 513-518, 2011.

27. Piao YR, Piao LZ, Zhu LH, et al: Prognostic value of T cell immunoglobulin mucin-3 in prostate cancer. Asian Pac J Cancer Prev 14: 3897-3901, 2013.

28. Cao Y, Zhou X, Huang X, et al: Tim-3 expression in cervical cancer promotes tumor metastasis. PLoS One 8: e53834, 2013.

29. Cai C, Wang L, Wu Z, et al: T-cell immunoglobulin- and mucin-domain-containing molecule 3 gene polymorphisms and renal cell carcinoma. DNA Cell Biol 31: 1285-1289, 2012.

30. Tong D, Zhou Y, Chen W, et al: T cell immunoglobulin- and mucin-domain-containing molecule 3 gene polymorphisms and susceptibility to pancreatic cancer. Mol Biol Rep 39: 9941-9946, 2012.

31. Tang D and Lotze MT: Tumor immunity times out: TIM-3 and HMGB1. Nat Immunol 13: 808-810, 2012. 